\title{
Les écritures de l'horreur en littératures africaines, B. Cyprien Bodo, M. Coulibaly, B. Kamagate (dir.)
}

\section{Emanuela Cacchioli}

\section{(2) OpenEdition}

1 Journals

\section{Edizione digitale}

URL: http://journals.openedition.org/studifrancesi/10076

DOI: 10.4000/studifrancesi. 10076

ISSN: 2421-5856

\section{Editore}

Rosenberg \& Sellier

\section{Edizione cartacea}

Data di pubblicazione: 1 août 2017

Paginazione: 408-410

ISSN: 0039-2944

\section{Notizia bibliografica digitale}

Emanuela Cacchioli, «Les écritures de l'horreur en littératures africaines, B. Cyprien Bodo, M. Coulibaly, B. Kamagate (dir.)», Studi Francesi [Online], 182 (LXI | II) | 2017, online dal 01 août 2017, consultato il 06 janvier 2021. URL: http://journals.openedition.org/studifrancesi/10076; DOI: https://doi.org/10.4000/ studifrancesi. 10076

Questo documento è stato generato automaticamente il 6 janvier 2021.

\section{(c) (1)}

Studi Francesi è distribuita con Licenza Creative Commons Attribuzione - Non commerciale - Non opere derivate 4.0 Internazionale. 


\title{
Les écritures de l'horreur en littératures africaines, B. Cyprien Bodo, M. Coulibaly, B. Kamagate (dir.)
}

\author{
Emanuela Cacchioli
}

\section{NOTIZIA}

Les écritures de l'horreur en littératures africaines, sous la direction de Bidy CYPRIEN BODO, Moussa COULIBALY, Bassidiki KAMAGATE, Paris, L'Harmattan, 2016, 443 pp.

1 È interessante il tema proposto nel volume pubblicato dalla casa editrice l'Harmattan. Trattandosi di una raccolta di saggi, la questione viene snocciolata da diverse prospettive e affrontata dal punto di vista del genere (si parla di romanzi, poesie, testi teatrali e racconti) e pluridisciplinare. I contributi ruotano attorno alla scrittura dell'orrore, intesa come rappresentazione letteraria della crudeltà, dell'efferatezza e delle sensazioni che tali atti provocano sulle vittime. Molti articoli rintracciano l'origine di tutti i mali nella Storia, con particolare riferimento al dominio del colonizzatore, alla neocolonizzazione, ai genocidi, sino al terrorismo dei giorni nostri. Quindi guerre e violenza, generalmente considerate indicibili o inenarrabili, trovano sempre di più negli ultimi decenni una forma di scrittura che le ingloba e ne dà conto attraverso racconti raccapriccianti e dal valore simbolico.

Le riflessioni si aprono con il contributo di Moussa couliBALY, Une fabrique impropre: "Elle sera de jaspe et de corail", un genre horrifique (pp. 9-19), che prende in esame il «chantroman» (p. 9) della scrittrice camerunense Werewere Liking. L'opera - che sfugge a ogni tipo di classificazione, in quanto mescolanza di generi e di stili - ha «une structure formelle déroutante qui suscite un dégoût» (p. 13). Il senso di repulsione nasce, dunque, dal caos compositivo, ma anche dalla rappresentazione quotidiana della violenza a cui il lettore si confronta grazie alla pagina scritta. Bidy Cyprien BODO, in L'impuissance acquise comme motif de l'horreur dans le roman africain: l'exemple de "Le jardin d'Adalou" de Josette 
Abondio (pp. 21-36), ci presenta l'orrore a partire da due prospettive differenti. Da un lato, esso è descritto come impulso incontrollabile, fatto di violenza, tortura e sofferenza, inflitti a una persona. Si tratta, in ultima analisi, di un comportamento rivelatore di una mostruosità insita nell'uomo. Dall'altro lato, si prende in considerazione la vittima e le conseguenze della crudeltà. Bassidiki KAMAGATÉ, con "Les indépendan-tristes": écriture et représentation de l'horreur chez Williams Sassine (pp. 37-50), analizza i meccanismi enunciativi e drammatici dell'orrore all'interno della pièce dello scrittore guineano. La ripugnanza è raccontata e mostrata nel rispetto della tradizione letteraria africana. Più che la banalità della violenza, Sassine esprime la sconfitta ideologica e filosofica delle indipendenze e di un'Africa che fatica a trovare l'equilibrio e il pieno sviluppo. L'atrocità mescolata all'ironia, per suscitare compassione e disgusto al tempo stesso, viene analizzata da Linda RASOAMANANA, in Quelle ironie innerve l'honneur: "Nerf de bouf" de Nassur Attoumani (pp. 51-74). Il romanzo mette in scena delle visioni da incubo che conducono il lettore al limite della sopportazione per raccontare l'atrocità della schiavitù. La sensazione di disgusto provocata dall'orrore della scrittura (forma) e nella scrittura (contenuto) in varie opere francofone della letteratura africana sono il focus dell'articolo di Pierre Suzanne EYENGA ONANA, Scénographie du sang des martyrs et figuration synecdochique de l'horreur dans l'imaginaire africain (pp. 75-99). La «postulation d'une culture de la paix fondée sur l'éthique de l'être-ensemble» (p. 98) ha lo scopo di spingere l'uomo verso una società dominata dalla tolleranza e dalla generosità. Ciò è possibile solo se l'individuo riesce a rompere il ciclo di violenza e di morte. Nella letteratura africana, l'orrore è legato all'esperienza storica della schiavitù, della colonizzazione e delle guerre tribali. A tal proposito, Lise MBA EKANI, con L'horreur en noir et rouge dans "Kouty, mémoire de sang" d'Aïda Mady Diallo (pp. 101-111), studia la riappropriazione dei codici del poliziesco come «réponse à la description de l'indicible pour une prise de conscience» (p. 109). Il contributo di Dégbéré Mélanie Coulibaly, L'écriture de la marge: une horreur narrative dans "La traversée du guerrier" de Jérôme Diegou Bailly (pp. 113-123) si focalizza sul margine inteso come tecnica che accompagna il testo, contribuisce alla sua elaborazione e prende in carico tutte le riflessioni al centro dell'opera. Il raccapricciante può costituire il materiale da cui si ricava il bello, come ci mostra K'Monti Jessé DIAMA in "La vie et demie" de Sony Labou Tansi: une poétisation de l'horreur (pp. 125-151). Per riuscire in una tale impresa è necessario procedere per accumulazione di immagini, di simboli e attraverso la ripetizione di sequenze. Viene ripreso il legame tra l'orrore e i fatti storici anche nell'articolo di Serigne SYLLA che segue: L'esthétique de l'horreur dans l'œeuvre poétique de Léopold Sédar Senghor (pp. 153-174). Nell'opera senghoriana la ripugnanza e l'indignazione sono prese in carico dai procedimenti retorici, dai campi lessicali, dal sistema enunciativo e dalle tecniche poetiche adottate dall'autore. Gratien LUKOGHO VAGHENI, invece, in Violences sexuelles et poésie R.D. Congolaise: le cas de Bestine Kazadi Ditabala (pp. 175-190), si occupa degli abusi sessuali perpetrati nell'Africa postcoloniale. Nonostante l'argomento sia ancora un tabù, rende bene conto dell'ossessione generata dall'eccesso immotivato della crudeltà. Il retaggio della storia è al centro anche di "L'intérieur de la nuit" de Léonora Miano ou l'écriture d'une nuit d'horreur (pp. 191-210) di Achille Carlos ZANGO. Lo studioso mette in luce come Miano abbia scelto di associare la notte alla violenza, in quanto entrambe condividono l'oscurità, reale o metaforica. Descrivere il terrore, tuttavia, ha uno scopo didattico, catartico, umanitario e memoriale. Mehani PATRIGEON, con Du fantôme au spectre: persistance d'une chair habitée dans "La vie et demie" de Sony Labou Tansi (pp. 211-222), ci offre un'ulteriore analisi del romanzo dello scrittore congolese. In questo 
caso si rintraccia l'estetica del grottesco e il ricorso alla dismisura e all'assurdo come degenerazione paranoica della politica. La «carnavalisation du récit» come strumento di denuncia è studiato da Willy KANGULUMBA MUNZENZA, in Écriture de la violence et carnavalisation du récit dans quelques romans africains francophones (pp. 223-247). Le opere analizzate si propongono di accusare con violenza i regimi neocoloniali. Baha Anicette Carolle AHOUAKAN, in Écriture et rhétorique de l'horreur dans "Les naufragés de l'intelligence" de J.-M. Ade Adiaffi (pp. 249-262), esamina il romanzo dello scrittore ivoriano, spaccato cupo della società in preda a un profondo malessere esistenziale. Con esso l'autore ricerca una satira sociale riferita alla Costa d'Avorio e, ancora una volta, la promozione dell'Africa contemporanea. Yao Constant ZEBIÉ, con L'horreur dans les écritures de violence: voyage au ccur des ténèbres (pp. 263-286) affronta il tema del genocidio e della ribellione. La scrittura dell'orrore cerca di comunicare l'incomunicabile, di nominare l'innominabile, di raccontare l'irraccontabile, per vincere gli incubi del reale. I bambini soldato e il loro difficile reintegro nella società sono lo spunto di riflessione di Fatoumata TOURÉ CISSÉ, in L'écriture de l'horreur de la guerre en Sierra-Leone dans "Chemin parcouru, mémoire d'un enfant-soldat" d'Ishmael Beah (pp. 287-297). Reo incolpevole dei suoi atti, il bambino chiede redenzione per crimini che ha commesso senza esserne il diretto responsabile. Il fatto che si tratti di eventi reali rende evidente come la guerra sia un gioco al massacro e le armi, anche se maneggiate da un ragazzo, siano sempre causa di morte, dolore e sofferenza. Evelyne NONGA, con Le terroriste dans "Les Funérailles" de Rachid Boudjedra, ou la mise en récit d'une abjection (pp. 301-324), affronta un'altra sfaccettatura dell'orrore: il terrorismo nelle sue molteplici forme, dagli attentati degli anni novanta a quelli che segnano la contemporaneità. L'inenarrabile e il rapporto tra la crudeltà e la guerra costituisce l'argomento del contributo successivo: L'horreur en scène dans le roman africain francophone: variations autour du réalisme et de l'indicible (pp. 325-335) di Didier Brou ANOH. La violenza fisica e quella simbolica sono legate l'una all'altra e permettono all'autrice di denunciare un universo sociale violento e ostile. $\grave{\mathrm{E}}$ quanto sostiene Cyrille Cédric NKO A BODIONG, in Les aspects de la violence dans la fiction horrifique de Leonora Miano (pp. 337-360). Bocar Aly PAM, dal canto suo, con Récit de guerre et esthétique de l'horreur à travers "Allah n'est pas obligé" (pp. 361-379), affronta con sguardo lucido i meccanismi che conducono l'Africa ai conflitti civili. Si nota come la creatività e l'ingegno umano siano impiegati per perpetrare violenza su altri individui. Il genocidio in Ruanda è il tema trattato da Pierre GOMEZ, in Moisson de crânes: la problématique d'un génocide programmé au Rwanda (pp. 381-393). Vengono ripercorse le tappe di questo crimine contro l'umanità nel romanzo di Abdourahman A. Waberi e il rapporto con altri olocausti come la Shoah o i crimini inflitti agli armeni e ai cambogiani. Bernard DE MEYER, in Pour une esthétique des ossements: la littérature postgénocide en Afrique francophone (pp. 395-413), tratta lo stesso argomento a partire dall'analisi di altri romanzi. Lo studioso tenta di fare luce e di portare a conoscenza il genocidio ruandese e lamenta la cortina di silenzio che si è posata su di esso. Chiude la raccolta Françoise UGochuKWU con Les contes igbo (Nigeria) et la gestion de l'horreur (pp. 415-434). Nell'articolo sono analizzati i racconti popolari in cui la comunità è alle prese con un mondo soprannaturale ambivalente che impone loro il confronto con la mostruosità.

3 Il volume si presenta come una serie di riflessioni eterogenee, in cui manca un filo conduttore o una visione unitaria che possa conferire sistematicità al discorso e individuarne linee di discussione. Peccato anche per la poca attenzione agli aspetti 
grafici. La collettanea è tuttavia interessante per la scelta di un tema originale e l'approccio fornito dai singoli contributi che permette comunque di farci un'idea della problematica e di alcune sue possibili sfaccettature. 